\title{
Performance Evaluation of Gray Scale Image using EZW and SPIHT Coding Schemes
}

\author{
Pooja Rawat \\ Scholar of M.tech, \\ GRD-IMT Dehradun
}

\author{
Ashish Nautiyal \\ Assistant Professor, \\ D.I.E.T Rishikesh
}

\author{
Swati Chamoli \\ Assistant Professor, \\ GRD-IMT Dehradun
}

\begin{abstract}
Digital wavelet transform based compression methods have higher compression rate with less amount of memory requirements, reversible and provide a better reconstructed images. In this paper execute image compression technique using EZW and SPIHT schemes. By using of different wavelet filters that is dmey, Symlets, Daubechies, Coiflets, reverse bi-orthogonal examine the compression performance. This method produces preserving most of the image information and the image is reproduced without degrading the image quality. Embedded zero tree wavelet is introduced by Shapiro and Amir Said introduced set partitioning in hierarchical trees. The best reconstructed images with better PSNR and minimum execution time provide by these techniques. Both techniques are compared by various parameters such as PSNR, CR, BPP, MSE \& execution time. The results of image compression algorithm analyzed using MATLAB software and wavelet toolbox.
\end{abstract}

\section{Keywords}

Image Compression, EZW, SPIHT, PSNR, CR, BPP, MSE, execution time

\section{INTRODUCTION}

By the advancement in Internet and multimedia (combination of media including video, still images, audio, text) technology, Image Compression is a technique to reduce the irrelevant information of an image. If the large volume of image data is needed to storage, higher transmitted bandwidth and large memory size is required too. So it is necessary for the transmission of large volume of data must be compressed by using image compression techniques. Image compression techniques make small the size of an image without reducing the quality of an image [1].

An Image is represented as 2-dimensional image consist of a set of pixels which has combination of information and redundancy. Information is an important part of an image that must be jammed permanently in its original form in order to translate the meaning or purpose of the data [1]. Redundancy contains repeated information and irrelevant which means the part of the signal that will not be observed by the human visual system. Three types of redundancy are presented here:

i) Spatial redundancy occurs because of the correlation between neighboring pixels.

ii) Spectral redundancy occurs because of the correlation between the color components. iii) Psycho-visual redundancy occurs because of the properties of the human visual system.

Image compression focuses on reducing the number of bits needed to represent an image by removing the spatial and spectral redundancies. The process of removal of spatial and spectral redundancy is often accomplished by the predictive coding or transforms coding. Quantization is the most important means of irrelevancy reduction [3].

\subsection{Classification of Image Compression}

Image compression can be understood as a method that takes an input image and generates a shorter representation of the image with less number of bits compared to that of original image. Image compression algorithms are classified into two categories - lossless and lossy.

1. Lossless: - Lossless compression reduces the overall image without removing any of the image detail. In this technique overall size of image will be compressed. Lossless compression methods are more appropriate for artificial images.Run-length encoding and entropy encoding are the methods for lossless image compression

2. Lossy: - Lossy compression removing image detail to one tenth of its original size with no visible changes to image quality [3], [8]. In lossy compression lost and absent information is endurable. Natural images are preferred by Lossy methods. Transform coding, DCT, Discrete wavelet transform are the most commonly used lossy compression methods.

Over the past few years, to achieve good compression ratio the image needs to be first transformed. This transformed image is to be stored and need to recover the image by using inverse transform and get back the original image. There are many transform schemes are used namely Fourier Transform (FT), Discrete Cosine Transform (DCT), Hadamard Transform, Wavelet transform and many more. Out of these transforms wavelet transform is the best compression technique that achieve higher compression ratio without reducing the image quality and mean square error [5]. Wavelet Transform is used in achieving better compression ratio, high peak signal to noise ratio to maintain the image quality and mean square error and execution time. 


\section{WAVELET AND WAVELET TRANSFORM}

\subsection{History of Wavelet}

Wavelets means small wave [1] used as a mathematical tool to extract the data into different frequency components which is suitable for compression [3]. Wavelets referred as a set of small waves [4] by Morlet and Grossmann, to improve the execution of image with enhanced execution time. There are many application of Wavelet include image compression, agitates, human vision, radar and earthquake prediction [2]. An engineer Morlet described wavelet of constant shape which is space localized as known as Morlet wavelets. Morlet and Grossman proved the reconstruction of wave could be possible from wavelet decomposition. After that the concept of orthogonality in wavelets introduced by Meyer. Orthogonality means when two functions are related with this equation

$$
\int_{-\infty}^{\infty} f_{1}(t) f_{2}(t) d t=0
$$

Then in 1986 Stephen Mallat student of Meyer added a powerful approach called multi resolution theory which includes sub band coding, quadrature mirror filtering [2]. In 1987 Ingrid Daubechies discovered a new class of wavelets which were not only orthogonal but can be implemented using simple and short digital filters [4].

\subsection{Wavelet filters}

According to I. Daubechies [4] wavelet transform can be implemented using simple and short digital filters. For multiresolution analysis, filters have some parameters for a particular region that is different to each other. A filter bank consists a number of filters with some parameters which contain different regions. Wavelet filters have two functions Scaling function and wavelet function. Scaling function $\phi(t)$ provide low frequency analysis and wavelet function $\psi(\mathrm{t})$ high frequency analysis [4]. In this paper there are five different type of filters dmey, db8, sym8, coif5, rbio5.5 are used in wavelet transform with their own functionality.

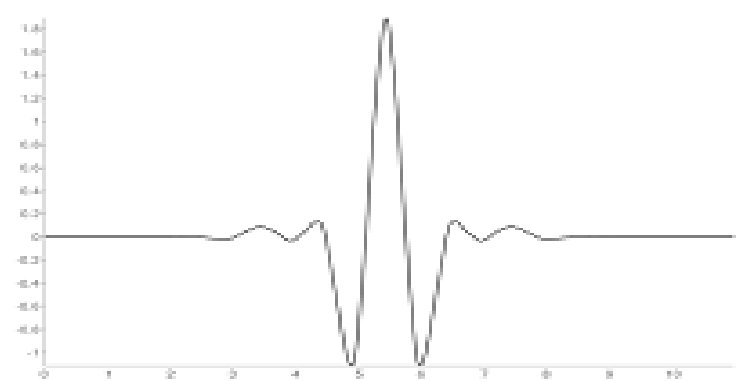

Fig2- (b) Wavelet function of rbio5.5 filter
Discrete Meyer (dmey): This wavelet filter is proposed by Y. Meyer. Symmetric, orthogonal, biorthogonal are the properties of dmey filter.

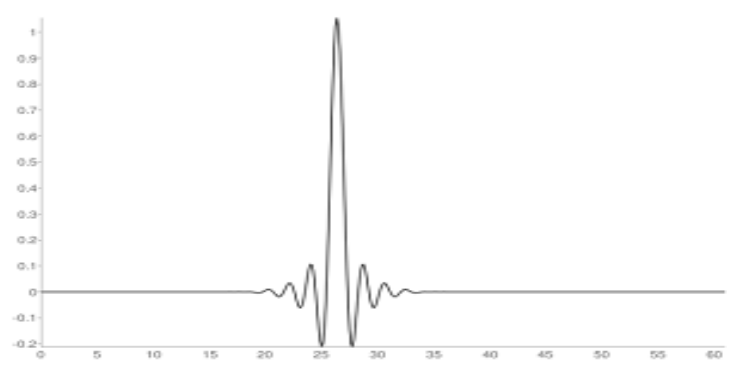

Fig 1- (a) Scaling function of dmey filter

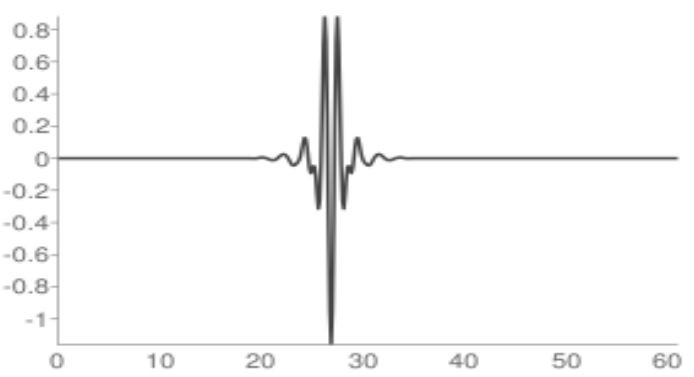

Fig1- (b) Wavelet function of dmey filter

Reverse biorthogonal (rbio): This is symmetric, not orthogonal, biorthogonal wavelet filter.

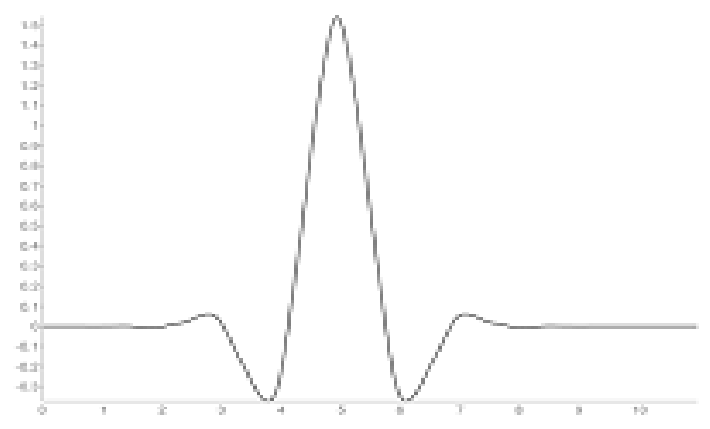

Fig 2- (a) Scaling function of rbio 5.5 filter 
Symlet (sym): Symlet filter is also known as least asymmetric filter in the orthogonal family.
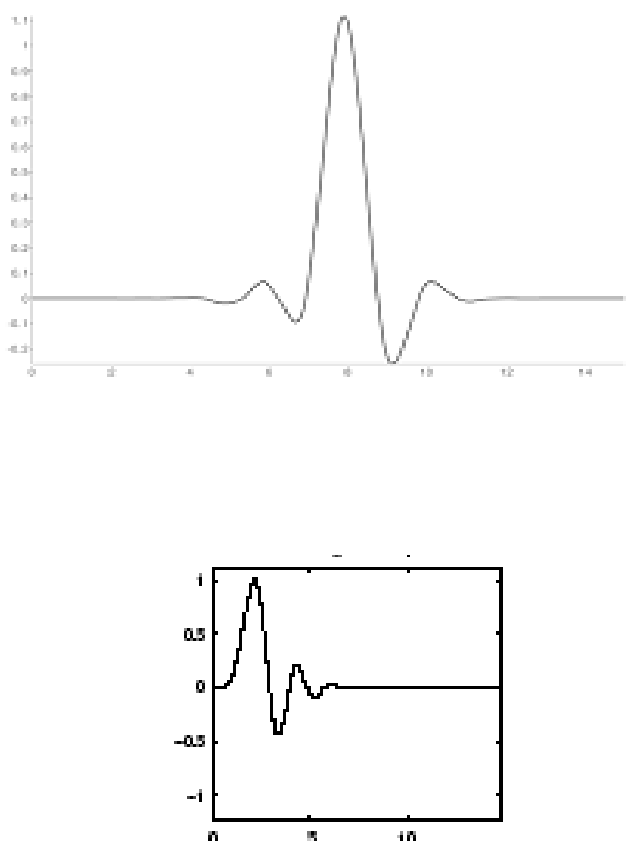

Fig 5- (a) Scaling function of db8 filter Fig 3- (a) Scaling function of sym8 filter

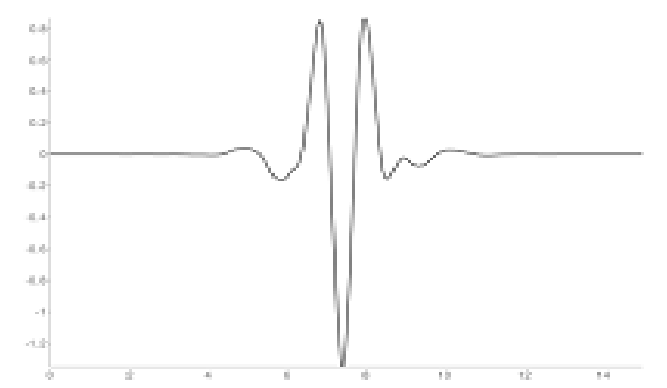

Fig3- (b) Wavelet function of sym8 filter

Coiflets (coif): This filter is near symmetric, orthogonal, bi-orthogonal wavelet filter.

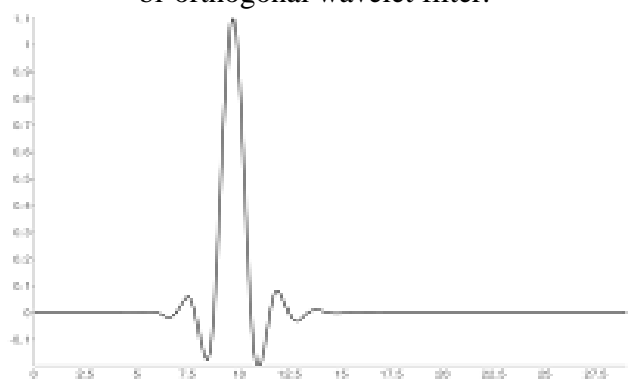

Fig 4- (a) Scaling function of coif5 filter

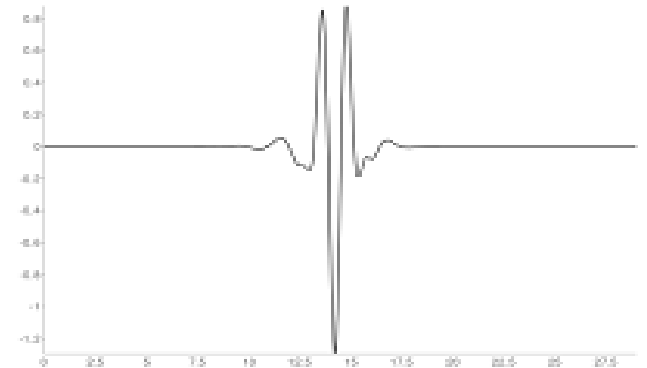

Fig4- (b) Wavelet function of coif5 filter

Daubechies (db): Properties of Daubechies filters are asymmetric, orthogonal, and bi-orthogonal.

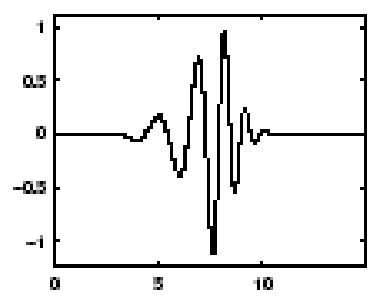

Fig5- (b) wavelet function of $\mathrm{db8}$ filter

\subsection{Discrete Wavelet Transform (DWT)}

Easier way to analyze many images, compress and transmit images, is wavelet transform than the Fourier transform. Wavelet transform analyzed different frequencies of an image at different resolution known as multi-resolution analysis [2]. To obtain a multiple level of decomposition discrete wavelet transform can be implemented by passing the signal through a repeated combination of low pass and high pass filters and down sampling operation on low pass branch outputs [5]. Discrete wavelet transform is applied both along rows and columns to obtain the approximation and details of images [4]. In Wavelet transform an image is first decomposed into four parts by critically sub sampling horizontal and vertical channels using sub band filters which is based on frequency sub bands. These sub bands represented as $L L_{n} H L_{n}, L H_{n}, H H_{n}$. These sub bands contains the information of approximation of Low pass filter over rows and low pass filter over columns. Horizontal High pass filter over rows and low pass filter over columns. Vertical Low pass filter over rows and high pass filter over columns and Diagonal Detail respectively. To obtained consisting of large particles LL sub band will further decomposed as shown the fig. 6

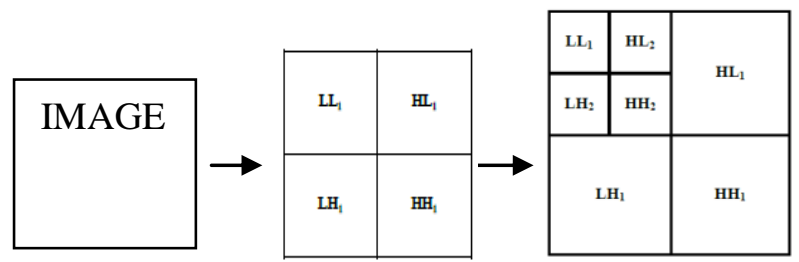




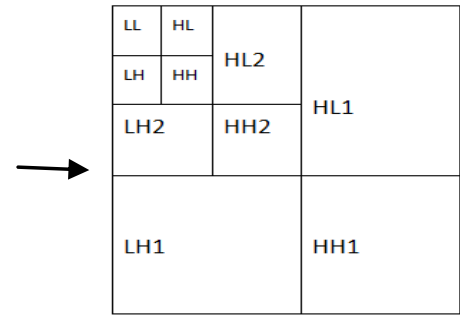

Fig 6: Representation of One level, two level and three level wavelet decomposition sub band

\section{EMBEDDED ZERO TREE WAVELET (EZW)}

The EZW image compression algorithm is introduced by $\mathrm{J}$. M. Shapiro [3], EZW encoding algorithm firstly divided an image into four sub bands using separable filters. The base of EZW is the parent -child dependencies of sub bands in wavelet transform. For the better performance of zero-tree coders parent child relationship responsible. Parent-child relationship means that the coefficient at the coarse scale is parent and at the finer scale all coefficients corresponding to the same spatial location is children. [3]

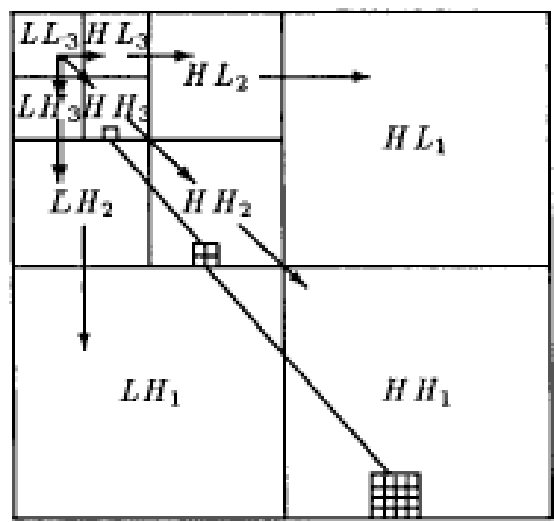

Fig 7: Parent- Child Dependencies

The EZW Algorithm consists following steps

\section{Step 1 Initialization}

This algorithm include the first step to determine the initial threshold level

$\mathrm{T}_{0}=2^{\log \mathrm{C}}{ }_{\max } \mid$

- Where Cmax is the maximum coefficient value.

Now wavelet coefficient matrix is scanned in order to find out the maximum value of the coefficient [7]. Maximum value of coefficients for any image occurs at the lowest frequency LL sub band. There are two passes which is used to code the image first pass is dominant pass and second one is subordinate pass.

1. LIP (list of insignificant pixel): LIP contains individual coefficient and have small magnitude than the threshold.

\section{Step 2 Dominant pass}

In dominant passes coefficients are compared to $\mathrm{T} 0$ and their significance map is made. Embedded Zero Tree Wavelet consists of four symbols which actually represents the significant map

1. Zero-tree root (t): If the root of wavelet coefficients tree and all the descendents lower than threshold value, known as zero tree root

2. Isolated zero $(\mathbf{z})$ : if the root of wavelet coefficient tree lower than the threshold value and some descendents are greater than threshold level then the root is known as isolated zero root

3. Significant positive/ negative (p/n): It is significant positive if the absolute value of the wavelet coefficient in the sub band decomposition of image is greater than the given threshold value, positive significant or negative significant according to their sign.

\section{Step 3 Subordinate pass}

In the subordinate list are refined the value of coefficient. This converts the dominant pass values into bit format if the value is greater than the current threshold then a 1 is sent, if less than the threshold a zero is sent. The current threshold is then divided by two and the process is repeated till the threshold doesn't reach the minimum bit value or zero. [7]

\section{SET PARTITIONING INHIERARCHAL TREES (SPIHT)}

SPIHT algorithm is improved version of EZW algorithm proposed by A. Said, Pearlman [6]. SPIHT algorithm is based on 3 concepts: Ordered bit plane progressive transmission, set partitioning sorting algorithm, spatial orientation trees [8]. SPIHT algorithm partitions the decomposed wavelet into significant and insignificant partitions based on the following function:

$$
S_{n}(\mathcal{T})= \begin{cases}1, & \max _{(i, j) \in \mathcal{T}}\left\{\left|c_{i, j}\right|\right\} \geq 2^{n}, \\ 0, & \text { otherwise }\end{cases}
$$

Thus SPIHT generates the best results of high PSNR at higher compression ratios than EZW due to its partitioning property which increases its compressive power. There are two passes the sorting pass and the refinement pass in this algorithm. Sorting pass is performed by the three type of list of pixels 
2. LIS (list of insignificant sets): It contains set of wavelet coefficient which are defined by tree structures, and found to have smaller magnitude than a threshold.

3. LSP (list of significant pixel): It contains the list of pixels which have larger magnitude than the threshold.

The SPIHT algorithm consists following steps: In this SPIHT algorithm there are different notations are used:

Off $(i, j)$ : set of coordinates of all offspring of node $(i, j)$

Des $(i, j)$ : set of coordinates of all descendants of node $(i, j)$ $\mathrm{H}(\mathrm{i}, \mathrm{j})$ : set of all tree roots or nodes in the highest pyramid level

L (i, j): Des (i, j) - Off (i,j) all descendants except the offspring

\section{Step 1: Initialization:}

$\mathrm{n}=\log 2(\max |\operatorname{coeff}|)$

LIP $=$ All elements in $\mathrm{H}$

LSP = Empty

LIS $=$ Des's of Roots

\section{Step2: Sorting pass:}

i) All pixels coordinate which remain in LIP are tested, if 1 then it is significant if 0 then it is not.

ii) After that significant output is transferred to the LSP having their sign.

iii) If Des $(i, j)$ is significant then partition it into $L(i, j)$ plus four single element sets of Des ( $i$, j) $€ \operatorname{Off}(i, j)$.

iv) If $L(i, j)$ is significant then partition it into four sets with Des (i, j) $€$ off (i, j).

\section{Step 3: Refinement pass:}

During the refinement pass, the nth most significant bit of the coefficients is output in the LSP. The value of $n$ is decreased by 1 and the steps 2 and 3 are repeated till $n=0$ is not occur.

\section{RESULT \& ANALYSIS}

In this paper we have implemented EZW and SPIHT coding schemes using various wavelets filters on a grayscale image i.e. chest X-Ray size (512X512). The experimental results are used to find the CR, BPP, PSNR values, MSE and execution time values for the reconstructed images. The result got by EZW \& SPIHT is shown in the following Tables 1-2. Table 1 represents the results of CR, BPP, and MSE \& PSNR by using EZW algorithm. Table 2 shows the result of different parameters for SPIHT Algorithms.
TABLE 1: Different Parameters Values of Different Wavelets for SPIHT Algorithm

\begin{tabular}{|l|l|l|l|l|l|}
\hline Wavelet & $\begin{array}{l}\text { PSNR } \\
\text { db }\end{array}$ & $\begin{array}{l}\text { MSE } \\
\text { db }\end{array}$ & BPP & $\begin{array}{l}\text { CR } \\
\text { \% }\end{array}$ & $\begin{array}{l}\text { Execution } \\
\text { time }\end{array}$ \\
\hline dmey & 41.8462 & 4.2507 & 1 & 87.5 & 70.9351 \\
\hline db8 & 41.7655 & 4.3305 & 1 & 87.5 & 75.2219 \\
\hline sym8 & 41.8304 & 4.2662 & 1 & 87.5 & 74.8031 \\
\hline coif5 & 41.8459 & 4.251 & 1 & 87.5 & 80.0989 \\
\hline rbio5.5 & 41.3528 & 4.7621 & 1 & 87.5 & 57.7587 \\
& & & & & \\
\hline
\end{tabular}

TABLE 2: Different Parameters Values of Different Wavelets for EZW Algorithm

\begin{tabular}{|c|c|c|c|c|c|}
\hline Wavelet & $\begin{array}{l}\text { PSNR } \\
\mathrm{db}\end{array}$ & $\begin{array}{l}\text { MSE } \\
\mathrm{db}\end{array}$ & BPP & $\begin{array}{l}\text { CR } \\
\%\end{array}$ & $\begin{array}{l}\text { Executi } \\
\text { on time }\end{array}$ \\
\hline dmey & 27.503 & $\begin{array}{l}115.554 \\
1\end{array}$ & $\begin{array}{l}0.8070 \\
7\end{array}$ & $\begin{array}{l}89.911 \\
7\end{array}$ & 27.6739 \\
\hline $\mathrm{db} 8$ & 26.386 & $\begin{array}{l}149.444 \\
1\end{array}$ & $\begin{array}{l}0.8190 \\
9\end{array}$ & $\begin{array}{l}89.761 \\
4\end{array}$ & 26.0223 \\
\hline sym8 & $\begin{array}{l}24.205 \\
3\end{array}$ & $\begin{array}{l}246.919 \\
2\end{array}$ & $\begin{array}{l}0.7940 \\
1\end{array}$ & $\begin{array}{l}90.074 \\
9\end{array}$ & 27.6836 \\
\hline coif5 & $\begin{array}{l}25.743 \\
5\end{array}$ & $\begin{array}{l}173.272 \\
7\end{array}$ & $\begin{array}{l}0.8590 \\
4\end{array}$ & 89.262 & 26.6274 \\
\hline rbio5.5 & $\begin{array}{l}27.468 \\
2\end{array}$ & $\begin{array}{l}116.483 \\
2\end{array}$ & $\begin{array}{l}0.7621 \\
8\end{array}$ & $\begin{array}{l}90.472 \\
8\end{array}$ & 25.9751 \\
\hline
\end{tabular}




\section{CONCLUSION}

In this paper, compared the results of two different waveletbased image compression techniques which are embedded zero tree wavelet (EZW) and set partitioning in hierarchical trees SPIHT. We are using different parameters such as peak signal to noise ratio, mean square error (MSE), and bit per pixel (bpp), compression ratio (CR) and execution time; the results are compared from the reconstructed image. According to analysis from the above table 1 and 2 we have analyzed less and fixed compression ratio, time for execution is high, MSE is low and PSNR is increased by using SPIHT coding whereas by using EZW coding with different filters, the high compression ratio at low bpp, execution time is less, lower value of PSNR but the mean square error (MSE) is high. From the results we found that in terms of PSNR and MSE the SPIHT algorithm is better than embedded zero tree wavelet algorithm. Various image coding algorithms in future can be analyzed for the betterment in the performance of those parameters.

\section{REFERENCES}

[1] Gonzalez, Rafael C., and Woods, Richard E., 2002 Digital Image Processing. Pearson Education, Englewood Cliffs.

[2] Soman, K.P., Ramchandran, K.I., 2005, "Insight into Wavelets - From Theory to Practice", Prentice Hall of India, Second Edition, pp. 6-9.

[3] Shapiro, J.M., 1993, "Embedded Image Coding Using Zero trees of Wavelet Coefficients" IEEE Trans. on Signal Processing, vol. 41, issue 12, pp 3445-3462.

[4] Daubechies I., 1992, Ten Lectures on Wavelets, SIAM.

[5] Sadashivappa G., AnandaBabu K.V.S., 2009, "Wavelet filters for image compression, an analytical study" ICGST-GVIP journal, volume (9), Issue (5), September 2009, ISSN: 1687-398X.

[6] Said A. and Pearlman W. A., 1996, "A new, fast and efficient image codec based on set-partitioning in hierarchical trees," IEEE Trans. Circuits Syst. Video Technol., vol. 6, pp. 243-250 (Jun. 1996).

[7] Raid A.M., .Khedr W.M, El-dosuky M. A. and Ahmed W., 2014, "Image Compression using Zero tree Wavelet", Signal \& Image Processing: An International Journal (SIPIJ) Vol.5, No.6.

[8] Dodla S., Y David SolmonRaju, Murali Mohan K.V., 2013, "Image Compression using Wavelet and SPIHT
Encoding Scheme", International Journal of Engineering Trends and Technology (IJETT) - Volume 4 Issue 9(Sep 2013)

[9] Nautiyal A., Tyagi I. and Pathela M., 2014, "PSNR Comparison of Lifting Wavelet Decomposed Modified SPIHT Coded Image with Normal SPIHT Coding" International Journal of Computer Applications 102(15):16-21.

[10] Tyagi I., Nautiyal A. and Pathela M., 2014, "Compression of Image using Enhanced EZW by Setting Detail Retaining Pass Number" International Journal of Computer Applications 96(2):37-44.

[11] Gandotra N., Bijalwan V., 2012, “coexistence model of zigbee \& IEEE $802.11 \mathrm{~b}$ (WLAN) in ubiquitous network environment", IJARCET, volume 1, Issue 4.

[12] Bijalwan V., Dr. Singh S., 2013, "analysis \& design of joint phy-mac model of ieee 802.15.4" IJSETR, volume 2, ISSUE 9.

[13] Bijalwan V., Pascual J., 2014, "KNN based Machine Learning Approach for Text \& Document Mining" International Journal of Database Theory and ApplicationVol.7,No.1(2014) ,pp61-70

[14] Nautiyal A., Tyagi I., Bijalwan V., Balodhi M., 2014, "Enhanced EZW Technique for Compression of Image by Setting Detail Retaining Pass Number", arXiv preprint arXiv:1407.3673,

[15] Balodhi M., Bijalwan V., Negi B., 2014, “'Zigbee\& IEEE 802.11 b (WLAN) coexistence in ubiquitous network environment", arXiv preprint arXiv: 1407.0462.

[16] Sati M., Vikash V., Bijalwan V., Kumari P., Raj M., 2014, “A Fault-Tolerant Mobile Computing Model Based On Scalable Replica”, IJIMAI.

[17] Bijalwan V., 2014, et al. "Machine learning approach for text and document mining." arXiv preprint arXiv: 1406.1580 .

[18] Gupta J.P., Singh N., Dixit P., Semwal V.B., Dubey S.R., 2013, "Human activity recognition using gait pattern."International Journal of Computer Vision and Image Processing (IJCVIP) 3.3 (2013): 31-53

[19] Bijalwan V., Balodhi M., Gusain A., 2013, "human emotion recognition using thermal image processing and eigen faces",

IJESR. 